\title{
A More General Maximal Bernstein-type Inequality
}

\author{
Péter Kevei * \\ MTA-SZTE Analysis and Stochastics Research Group \\ Bolyai Institute, Aradi vértanúk tere 1, 6720 Szeged, Hungary \\ e-mail: kevei@math.u-szeged.hu \\ David M. Mason ${ }^{\dagger}$ \\ University of Delaware \\ 213 Townsend Hall, Newark, DE 19716, USA \\ e-mail: davidm@udel.edu
}

May 24, 2012

\begin{abstract}
We extend a general Bernstein-type maximal inequality of Kevei and Mason (2011) for sums of random variables.

Keywords: Bernstein inequality, dependent sums, maximal inequality, mixing, partial sums.

AMS Subject Classificiation: MSC 60E15; MSC 60F05; MSC $60 \mathrm{G} 10$.
\end{abstract}

\section{Introduction}

Let $X_{1}, X_{2}, \ldots$ be a sequence of random variables, and for any choice of $1 \leq k \leq l<\infty$ we denote the partial sum $S(k, l)=\sum_{i=k}^{l} X_{i}$, and define $M(k, l)=\max \{|S(k, k)|, \ldots,|S(k, l)|\}$. It turns out that under a variety of assumptions the partial sums $S(k, l)$ will satisfy a generalized Bernstein-type inequality of the following form: for suitable constants $A>0, a>0, b \geq 0$ and $0<\gamma<2$ for all $m \geq 0, n \geq 1$ and $t \geq 0$,

$$
\mathbf{P}\{|S(m+1, m+n)|>t\} \leq A \exp \left\{-\frac{a t^{2}}{n+b t^{\gamma}}\right\} .
$$

Kevei and Mason [2] provide numerous examples of sequences of random variables $X_{1}, X_{2}, \ldots$, that satisfy a Bernstein-type inequality of the form (1.1). They show, somewhat unexpectedly, without any additional assumptions, a modified version of it also holds for $M(1+m, n+m)$ for all $m \geq 0$ and $n \geq 1$. Here is their main result.

Theorem 1.1. Assume that for constants $A>0, a>0, b \geq 0$ and $\gamma \in(0,2)$, inequality (1.1) holds for all $m \geq 0, n \geq 1$ and $t \geq 0$. Then for every $0<c<a$ there exists a $C>0$ depending only on $A, a, b$ and $\gamma$ such that for all $n \geq 1, m \geq 0$ and $t \geq 0$,

$$
\mathbf{P}\{M(m+1, m+n)>t\} \leq C \exp \left\{-\frac{c t^{2}}{n+b t^{\gamma}}\right\} .
$$

\footnotetext{
*Supported by the TAMOP-4.2.1/B-09/1/KONV-2010-0005 project.

${ }^{\dagger}$ Research partially supported by NSF Grant DMS-0503908.
} 
There exists an interesting class of Bernstein-type inequalities that are not of the form (1.1). Here are two motivating examples.

Example 1. Assume that $X_{1}, X_{2}, \ldots$, is a stationary Markov chain satisfying the conditions of Theorem 6 of Adamczak [1] and let $f$ be any bounded measurable function such that $E f\left(X_{1}\right)=$ 0 . His theorem implies that for some constants $D>0, d_{1}>0$ and $d_{2}>0$ for all $t \geq 0$ and $n \geq 1$,

$$
P\left\{\left|S_{n}(f)\right| \geq t\right\} \leq D^{-1} \exp \left(-\frac{D t^{2}}{n d_{1}+t d_{2} \log n}\right),
$$

where $S_{n}(f)=\sum_{i=1}^{n} f\left(X_{i}\right)$, and $D / d_{1}$ is related to the limiting variance in the central limit theorem.

Example 2. Assume that $X_{1}, X_{2}, \ldots$, is a strong mixing sequence with mixing coefficients $\alpha(n), n \geq 1$, satisfying for some $d>0, \alpha(n) \leq \exp (-2 d n)$. Also assume that $E X_{i}=0$ and for some $M>0,\left|X_{i}\right| \leq M$, for all $i \geq 1$. Theorem 2 of Merlevède, Peligrad and Rio [4] implies that for some constant $D>0$ for all $t \geq 0$ and $n \geq 1$,

$$
P\left\{\left|S_{n}\right| \geq t\right\} \leq D \exp \left(-\frac{D t^{2}}{n v^{2}+M^{2}+t M(\log n)^{2}}\right),
$$

where $S_{n}=\sum_{i=1}^{n} X_{i}$ and $v^{2}=\sup _{i>0}\left(\operatorname{Var}\left(X_{i}\right)+2 \sum_{j>i}\left|\operatorname{cov}\left(X_{i}, X_{j}\right)\right|\right)$.

The purpose of this note to establish the following extended version of Theorem 1.1 that will show that a maximal version of inequalities (1.3) and (1.4) also holds.

Theorem 1.2. Assume that there exist constants $A>0$ and $a>0$ and a sequence of nondecreasing non-negative functions $\left\{g_{n}\right\}_{n \geq 1}$ on $(0, \infty)$, such that for all $t>0$ and $n \geq 1, g_{n}(t) \leq$ $g_{n+1}(t)$ and for all $0<\rho<1$

$$
\lim _{n \rightarrow \infty} \inf \left\{\frac{t^{2}}{g_{n}(t) \log t}: g_{n}(t)>\rho n\right\}=\infty
$$

where the infimum of the empty set is defined to be infinity, such that for all $m \geq 0, n \geq 1$ and $t \geq 0$,

$$
P\{|S(m+1, m+n)|>t\} \leq A \exp \left\{-\frac{a t^{2}}{n+g_{n}(t)}\right\} .
$$

Then for every $0<c<a$ there exists a $C>0$ depending only on $A, a$ and $\left\{g_{n}\right\}_{n \geq 1}$ such that for all $n \geq 1, m \geq 0$ and $t \geq 0$,

$$
P\{M(m+1, m+n)>t\} \leq C \exp \left\{-\frac{c t^{2}}{n+g_{n}(t)}\right\} .
$$

Note that condition (1.5) trivially holds when the functions $g_{n}$ are bounded, since the corresponding sets are empty sets. However, in the interesting cases $g_{n}$ 's are not bounded, and in this case the condition basically says that $g_{n}(t)$ increases slower than $t^{2}$.

Essentially the same proof shows that the statement of Theorem 1.2 remains true if in the numerator of (1.6) and (1.7) the function $t^{2}$ is replaced by a regularly varying function at infinity $f(t)$ with a positive index. In this case the $t^{2}$ in condition (1.5) must be replaced by $f(t)$. Since we do not know any application of a result of this type, we only mention this generalization. 
Proof. Choose any $0<c<a$. We prove our theorem by induction on $n$. Notice that by the assumption, for any integer $n_{0} \geq 1$ we may choose $C>A n_{0}$ to make the statement true for all $1 \leq n \leq n_{0}$. This remark will be important, because at some steps of the proof we assume that $n$ is large enough. Also since the constants $A$ and $a$ in (1.6) are independent of $m$, we can without loss of generality assume $m=0$.

Assume the statement holds up to some $n \geq 2$. (The constant $C$ will be determined in the course of the proof.)

Case 1. Fix a $t>0$ and assume that

$$
g_{n+1}(t) \leq \alpha n
$$

for some $0<\alpha<1$ be specified later. (In any case, we assume that $\alpha n \geq 1$.) Using an idea of [5], we may write for arbitrary $1 \leq k<n, 0<q<1$ and $p+q=1$ the inequality

$$
\begin{aligned}
P\{M(1, n+1)>t\} \leq & P\{M(1, k)>t\}+P\{|S(1, k+1)|>p t\} \\
& +P\{M(k+2, n+1)>q t\} .
\end{aligned}
$$

Let

$$
u=\frac{n+g_{n+1}(q t)-q^{2} g_{n+1}(t)}{1+q^{2}} .
$$

Note that $u \leq n-1$ if $0<\alpha<1$ is chosen small enough depending on $q$, for $n$ large enough. Notice that

$$
\frac{t^{2}}{u+g_{n+1}(t)}=\frac{q^{2} t^{2}}{n-u+g_{n+1}(q t)}
$$

Set

$$
k=\lceil u\rceil .
$$

Using the induction hypothesis and (1.6), keeping in mind that $1 \leq k \leq n-1$, we obtain

$$
\begin{aligned}
P\{M(1, n+1)>t\} \leq & C \exp \left\{-\frac{c t^{2}}{k+g_{k}(t)}\right\}+A \exp \left\{-\frac{a p^{2} t^{2}}{k+1+g_{k+1}(p t)}\right\} \\
& +C \exp \left\{-\frac{c q^{2} t^{2}}{n-k+g_{n-k}(q t)}\right\} \\
\leq & C \exp \left\{-\frac{c t^{2}}{k+g_{n+1}(t)}\right\}+A \exp \left\{-\frac{a p^{2} t^{2}}{k+1+g_{n+1}(p t)}\right\} \\
& +C \exp \left\{-\frac{c q^{2} t^{2}}{n-k+g_{n+1}(q t)}\right\}
\end{aligned}
$$

Notice that we chose $k$ to make the first and third terms in (1.11) almost equal, and since by (1.10)

$$
\frac{t^{2}}{k+g_{n+1}(t)} \leq \frac{q^{2} t^{2}}{n-k+g_{n+1}(q t)}
$$

the first term is greater than or equal to the third.

First we handle the second term in formula (1.11), showing that whenever $g_{n+1}(t) \leq \alpha n$,

$$
\exp \left\{-\frac{a p^{2} t^{2}}{k+1+g_{n+1}(p t)}\right\} \leq \exp \left\{-\frac{c t^{2}}{n+1+g_{n+1}(t)}\right\} \text {. }
$$


For this we need to verify that for $g_{n+1}(t) \leq \alpha n$,

$$
\frac{a p^{2}}{k+1+g_{n+1}(p t)}>\frac{c}{n+1+g_{n+1}(t)},
$$

which is equivalent to

$$
a p^{2}\left(n+1+g_{n+1}(t)\right)>c\left(k+1+g_{n+1}(p t)\right) .
$$

Using that

$$
k=\lceil u\rceil \leq u+1=1+\frac{1}{1+q^{2}}\left[n+g_{n+1}(q t)-q^{2} g_{n+1}(t)\right],
$$

it is enough to show

$$
\begin{gathered}
n\left(a p^{2}-\frac{c}{1+q^{2}}\right)+a p^{2}-2 c \\
+\left[g_{n+1}(t) a p^{2}-g_{n+1}(p t) c-\frac{c}{1+q^{2}}\left(g_{n+1}(q t)-q^{2} g_{n+1}(t)\right)\right]>0 .
\end{gathered}
$$

Note that if the coefficient of $n$ is positive, then we can choose $\alpha$ in (1.8) small enough to make the above inequality hold. So in order to guarantee (1.12) (at least for large $n$ ) we only have to choose the parameter $p$ so that $a p^{2}-c>0$, which implies that

$$
a p^{2}-\frac{c}{1+q^{2}}>0
$$

holds, and then select $\alpha$ small enough, keeping mind that we assume $\alpha n \geq 1$ and $k \leq n-1$.

Next we treat the first and third terms in (1.11). Because of the remark above, it is enough to handle the first term. Let us examine the ratio of $C \exp \left\{-c t^{2} /\left(k+g_{n+1}(t)\right)\right\}$ and $C \exp \left\{-c t^{2} /(n+\right.$ $\left.\left.1+g_{n+1}(t)\right)\right\}$. Notice again that since $u+1 \geq k$, the monotonicity of $g_{n+1}(t)$ and $g_{n+1}(t) \leq \alpha n$ implies

$$
\begin{aligned}
n+1-k & \geq n-u=n-\frac{n+g_{n+1}(q t)-q^{2} g_{n+1}(t)}{1+q^{2}} \\
& \geq \frac{q^{2} n-\left(1-q^{2}\right) g_{n+1}(t)}{1+q^{2}} \\
& \geq n \frac{q^{2}-\alpha\left(1-q^{2}\right)}{1+q^{2}} \\
& =: c_{1} n .
\end{aligned}
$$

At this point we need that $0<c_{1}<1$. Thus we choose $\alpha$ small enough so that

$$
q^{2}-\alpha\left(1-q^{2}\right)>0
$$

Also we get using $g_{n+1}(t) \leq \alpha n$ the bound

$$
\left(n+1+g_{n+1}(t)\right)\left(k+g_{n+1}(t)\right) \leq 2 n^{2}(1+\alpha)^{2}=: c_{2} n^{2},
$$

which holds if $n$ large enough. Therefore, we obtain for the ratio

$$
\exp \left\{-c t^{2}\left(\frac{1}{k+g_{n+1}(t)}-\frac{1}{n+1+g_{n+1}(t)}\right)\right\} \leq \exp \left\{-\frac{c c_{1} t^{2}}{c_{2} n}\right\} \leq \mathrm{e}^{-1}
$$


whenever $c c_{1} t^{2} /\left(c_{2} n\right) \geq 1$, that is $t \geq \sqrt{c_{2} n /\left(c c_{1}\right)}$. Substituting back into (1.11), for $t \geq$ $\sqrt{c_{2} n /\left(c c_{1}\right)}$ and $g_{n+1}(t) \leq \alpha n$ we obtain

$$
\begin{gathered}
P\{M(1, n+1)>t\} \\
\leq\left(\frac{2}{\mathrm{e}} C+A\right) \exp \left\{-c t^{2} /\left(n+1+g_{n+1}(t)\right)\right\} \leq C \exp \left\{-c t^{2} /\left(n+1+g_{n+1}(t)\right)\right\},
\end{gathered}
$$

where the last inequality holds for $C>A \mathrm{e} /(\mathrm{e}-2)$.

Next assume that $t<\sqrt{c_{2} n /\left(c c_{1}\right)}$. In this case choosing $C$ large enough we can make the bound $>1$, namely

$$
C \exp \left\{-\frac{c t^{2}}{n+1+g_{n+1}(t)}\right\} \geq C \exp \left\{-\frac{c c_{2} n}{c c_{1} n}\right\}=C \mathrm{e}^{-c_{2} / c_{1}} \geq 1
$$

if $C>\mathrm{e}^{c_{2} / c_{1}}$.

Case 2. Now we must handle the case $g_{n+1}(t)>\alpha n$. Here we apply the inequality

$$
P\{M(1, n+1)>t\} \leq P\{M(1, n)>t\}+P\{|S(1, n+1)|>t\} .
$$

Using assumption (1.6) and the induction hypothesis, we have

$$
\begin{aligned}
P\{M(1, n+1)>t\} & \leq C \exp \left\{-\frac{c t^{2}}{n+g_{n}(t)}\right\}+A \exp \left\{-\frac{a t^{2}}{n+1+g_{n+1}(t)}\right\} \\
& \leq C \exp \left\{-\frac{c t^{2}}{n+g_{n+1}(t)}\right\}+A \exp \left\{-\frac{a t^{2}}{n+1+g_{n+1}(t)}\right\} .
\end{aligned}
$$

We will show that the right side $\leq C \exp \left\{-c t^{2} /\left(n+1+g_{n+1}(t)\right)\right\}$. For this it is enough to prove

$$
\begin{aligned}
& \exp \left\{-c t^{2}\left(\frac{1}{n+g_{n+1}(t)}-\frac{1}{n+1+g_{n+1}(t)}\right)\right\} \\
& +\frac{A}{C} \exp \left\{-\frac{t^{2}(a-c)}{n+1+g_{n+1}(t)}\right\} \leq 1 .
\end{aligned}
$$

Using the bound following from $g_{n+1}(t)>\alpha n$ and recalling that $\alpha n \geq 1$ and $0<\alpha<1$, we get

$$
\frac{t^{2}}{\left(n+g_{n+1}(t)\right)\left(n+1+g_{n+1}(t)\right)} \geq \frac{\alpha^{2} t^{2}}{(1+\alpha)(1+2 \alpha) g_{n+1}(t)^{2}}=: c_{3} \frac{t^{2}}{g_{n+1}(t)^{2}},
$$

and

$$
\frac{t^{2}(a-c)}{n+1+g_{n+1}(t)} \geq \frac{t^{2}}{g_{n+1}(t)} \frac{\alpha(a-c)}{1+2 \alpha}=: \frac{t^{2}}{g_{n+1}(t)} c_{4} .
$$

Choose $\delta>0$ so small such that $0<x \leq \delta$ implies $\mathrm{e}^{-c c_{3} x^{2}} \leq 1-\frac{c c_{3}}{2} x^{2}$. For $t / g_{n+1}(t) \geq \delta$ the left-hand side of (1.15) is less then

$$
\mathrm{e}^{-c c_{3} \delta^{2}}+\frac{A}{C},
$$

which is less than 1 , for $C$ large enough. 
For $t / g_{n+1}(t) \leq \delta$ by the choice of $\delta$ the left-hand side of (1.15) is less then

$$
1-\frac{c c_{3}}{2} \frac{t^{2}}{g_{n+1}(t)^{2}}+\frac{A}{C} \exp \left\{-\frac{t^{2}}{g_{n+1}(t)} c_{4}\right\}
$$

which is less than 1 if

$$
\frac{c c_{3}}{2} \frac{t^{2}}{g_{n+1}(t)^{2}}>\frac{A}{C} \exp \left\{-\frac{t^{2}}{g_{n+1}(t)} c_{4}\right\} .
$$

By (1.5), for any $0<\eta<1$ and all large enough $n, g_{n+1}(t) 1\left\{g_{n+1}(t)>\alpha n\right\} \leq \eta t^{2}$, so that for all large $n$, whenever $g_{n+1}(t)>\alpha n$, we have

$$
\frac{t^{2}}{g_{n+1}(t)^{2}} \geq t^{-2}
$$

and again by (1.5) for all large $n$, whenever $g_{n+1}(t)>\alpha n, t^{2} / g_{n+1}(t) \geq\left(3 / c_{4}\right) \log t$. Therefore for all large $n$, whenever $g_{n+1}(t) \alpha n$,

$$
\exp \left\{-\frac{t^{2}}{g_{n+1}(t)} c_{4}\right\} \leq t^{-3}
$$

which is smaller than $t^{-2} \frac{C c c_{3}}{2 A}$, for $t$ large enough, i.e. for $n$ large enough. The proof is complete.

By choosing $g_{n}(t)=b t^{\gamma}$ for all $n \geq 1$ we see that Theorem 1.2 gives Theorem 1.1 as a special case. Also note that Theorem 1.2 remains valid for sums of Banach space valued random variables with absolute value $|\cdot|$ replaced by norm $\|\cdot\|$. Theorem 1.2 permits us to derive the following maximal versions of inequalities (1.3) and (1.4).

Application 1. In Example 1 one readily checks that the assumptions of Theorem 1.2 are satisfied with $A=D^{-1}$ and $a=D / d_{1}$

$$
g_{n}(t)=\left(\frac{t d_{2}}{d_{1}}\right) \log n
$$

We get the maximal version of inequality (1.3) holding for any $0<c<1$ and all $n \geq 1$ and $t>0$

$$
P\left\{\left|\max _{1 \leq m \leq n} S_{n}(f)\right| \geq t\right\} \leq C \exp \left(-\frac{c D t^{2}}{n d_{1}+t d_{2} \log n}\right),
$$

for some constant $C \geq D^{-1}$ depending on $c, D^{-1}, D / d_{1}$ and $\left\{g_{n}\right\}_{n \geq 1}$.

Application 2. In Example 2 one can verify that the assumptions of the Theorem 1.2 hold with $A=D$ and $a=D / v^{2}$ and

$$
g_{n}(t)=\frac{M^{2}}{v^{2}}+\left(\frac{t M}{v^{2}}\right)(\log n)^{2},
$$

which leads to the maximal version of inequality (1.4) valid for any $0<c<1$ and all $n \geq 1$ and $t>0$

$$
P\left\{\max _{1 \leq m \leq n}\left|S_{m}\right| \geq t\right\} \leq C \exp \left(-\frac{c D t^{2}}{n v^{2}+M^{2}+t M(\log n)^{2}}\right)
$$


for some constant $C \geq D$ depending on $c, D / v^{2}$ and $\left\{g_{n}\right\}_{n \geq 1}$. See Corollary 24 of Merlevède and Peligrad [3] for a closely related inequality that holds for all $n \geq 2$ and $t>K \log n$ for some $K>0$.

Remark There is a small oversight in the published version of the Kevei and Mason paper. Here are the corrections that fix it.

1. Page 1057, line -9: Replace " $1 \leq k \leq n$ " by " $1 \leq k<n$ ".

2. Page 1057, line -7: Replace this line with

$\leq \mathbf{P}\{M(1, k)>t\}+\mathbf{P}\{S(1, k+1)>p t\}+\mathbf{P}\{M(k+2, n+1)>q t\}$.

3. Page 1058: Replace " $k+b p^{\gamma} t^{\gamma}$ " by " $k+1+b p^{\gamma} t^{\gamma}$ " in equations (2.4) and (2.5), as well as in line -13 .

4. Page 1058: Replace " $a p^{2}-c$ " by " $a p^{2}-2 c$ " in line -9 .

\section{Acknowledgment}

We thank a referee for a careful reading of the manuscript and a number of useful comments.

\section{References}

[1] R. Adamczak, A tail inequality for suprema of unbounded empirical processes with applications to Markov chains. Electron. J. Probab. 13 (2008), 1000-1034.

[2] P. Kevei and D.M. Mason, A note on a maximal Bernstein inequality. Bernoulli 17 (2011), 1054-1062.

[3] F. Merlevède and M. Peligrad, Rosenthal-type inequalities for the maximum of partial sums of stationary processes and examples. Ann. Probab. To appear.

[4] F. Merlevède, M. Peligrad, M. and E. Rio, Bernstein inequality and moderate deviations under strong mixing conditions. In: High Dimensional Probability V: The Luminy Volume, C. Houdré, V. Koltchinskii, D. M. Mason and M. Peligrad, eds., (Beachwood, Ohio, USA: IMS, 2009), 273-292.

[5] F.A. Móricz, R.J. Serfling and W.F. Stout, Moment and probability bounds with quasisuperadditive structure for the maximum partial sum. Ann. Probab. 10 (1982), 1032-1040. 